\title{
Signifikante Reduktion der Schmerzintensität und Verbesserung der Schlafqualität
}

Fragestellung: Diese Studie untersuchte in einer Metaanalyse aus neun Studien mit insgesamt 2.056 Patienten die Wirksamkeit von Pregabalin zur Behandlung der schmerzhaften diabetischen Polyneuropathie.

Hintergrund: Von den Patienten, die unter einer schmerzhaften diabetischen Neuropathie leiden, haben $10-20 \%$ behandlungsbedürftige Schmerzen. Mehrere Studien zeigen, dass Pregabalin diese Schmerzen signifikant zu lindern vermag; in einzelnen Studien ist allerdings kein Nutzen zu erkennen. Die vorliegende Metaanalyse hatte die Zielvorgabe, hier Klarheit zu schaffen.

Zhang SS, Wu Z, Zhang LC et al. Efficacy and safety of pregabalin for treating painful diabetic peripheral neuropathy: a meta-analysis. Acta Anaesthesiologica Scandinavica 2015; 59: $78-84$
Patienten und Methodik: Für die Metaanalyse wurden neun randomisierte, placebokontrollierte, doppelblinde Studien mit insgesamt 2.056 Patienten, die unter schmerzhafter diabetischer Neuropathie litten, ausge- wählt. Primäre Outcome-Variablen waren die durchschnittlichen Schmerzstärken sowie die Anzahl der Patienten, die eine Reduktion der Schmerzintensität von mehr als 50\% angaben. Die Analyse führten zwei unabhängige Untersucher unter anderem nach den Kriterien der Cochrane Collaboration durch. Es erfolgte die Datenextraktion anhand zahlreicher Variablen wie Schmerzstärke, Schlafqualität und das Auftreten von Nebenwirkungen.

Ergebnisse: Alle Studien die ausgewählt wurden, waren von hoher Qualität. Die mittleren Schmerzstärken waren in der Verumgruppe signifikant erniedrigt, ebenso die Anzahl der Patienten mit mehr als $50 \%$ Schmerzreduktion. Scores zur Schlafqualität zeigten sich in der Verumgruppe ebenfalls besser. Die Abbruchrate der Medikation war gegenüber Placebo erhöht. Dies betraf vornehmlich die höhere Dosisgruppe (300 mg vs. $600 \mathrm{mg}$ ).

Schlussfolgerungen: Pregabalin zeigte eine effektive Reduktion neuropathischer Schmerzen und verbessert Begleiterscheinungen der Erkrankung, die zur verbesserten Lebensqualität beitragen können.

\section{- Kommentar von Tim Hagenacker, Essen}

\section{Keine Änderung des Therapiekonzeptes}

Diese Metaanalyse zeigt noch einmal, dass Pregabalin zur Behandlung der schmerzhaften diabetischen Polyneuropathie gegenüber einem Placebo überlegen ist. Dabei zeigte sich sowohl eine signifikante Reduktion der Schmerzintensität als auch eine Verbesserung der Schlafqualität. Das gegenüber Placebo vermehrte Auftreten von Nebenwirkungen ist wenig überra-

Weitere Infos auf springermedizin.de

Kausale und symptomatische Therapie von

Polyneuropathien

Voraussetzung für die zielgerichtete Behandlung einer Polyneuropathie ist die Ursachensuche, da je nach Ätiologie verschiedene kausale Therapieoptionen zur Verfügung stehen können. Eine weitere wichtige Säule der Therapie stellt die symptomatische Behandlung polyneuropathischer Symptome dar, etwa die neuropathischer Schmerzen (5338320). schend. Auch wenn diese Metaanalyse die positive Wirkung von Pregabalin noch einmal klar demonstriert, ist der Wert dieser Analyse dennoch bescheiden. So ist Pregabalin bereits seit einiger Zeit Bestandteil der Leitlinie der European Federation of Neurological Societies (EFNS) zur Behandlung neuropathischer Schmerzen und findet in der täglichen Praxis schon lange breite Anwendung und das nicht nur bei Neurologen, sondern auch bei Diabetologen. Die Studie zeigt aber in der tabellarischen Darstellung, vielleicht unfreiwillig, etwas ganz wesentliches: Die meisten Studien beobachten nur über zwölf Wochen. Was also nun gefragt ist, ist die langfristige Wirksamkeit und welche Kombinationen mit anderen Medikamentengruppen synergistische Effekte zeigen können.

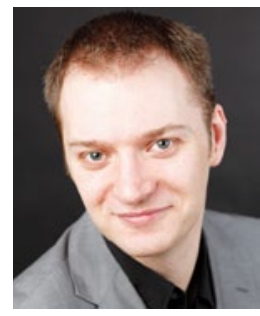

PD Dr. med. Tim Hagenacker, Essen

Klinik für Neurologie,

Universitätsklinikum Essen

E-Mail: tim.hagenacker@uk-essen.de 